\title{
Insight in the Kinenoetic Field
}

Frédéric Vallée-Tourangeau

Kingston University

Address correspondence to Frédéric Vallée-Tourangeau, Department of Psychology, Kingston University, Penrhyn Road, Kingston upon Thames, UNITED KINGDOM, KT1 2EE; f.vallee-tourangeau@kingston.ac.uk. I thank Lambros Malafouris, Paul March, Wendy Ross and Gaëlle Vallée-Tourangeau for comments on a previous version of this chapter. 


\begin{abstract}
Insight problems offer an interesting tool to observe how new ideas are developed to solve simple but vexing problems. Research typically proceeds with so-called second order procedures where participants are prevented from interacting with the world, forcing them to find a solution through mental processes alone. In contrast, when participants can interact with the environment they think with and through the world by building a physical model of the solution. Properly instrumentalized, such a first order procedure enables the granular tracing of the changes to the model of the solution. These changes reflect the co-constitutive coupling of thoughts and object, or object-thought mutualities. These mutualities are recovered with the mapping of the kinenoetic field, a term coined to capture the emergence of new ideas through the spatio-temporal changes to a physical object, namely the dynamic evolution of the model of the solution. We contrast the explanatory promises and challenges that arise from a programme of research based on second order problem solving with those from a programme that explores first order thinking.
\end{abstract}




\section{Insight in the Kinenoetic Field}

The disciplinary exigencies of cognitive psychology qua science of the mind guide and constrain the nature of the research enterprise. The ontological keystone of this enterprise is the enduring notion that mental processes are the prime drivers of the construction of knowledge, the primary cause of the origin of new ideas. Coupled to this notion is the apparently inescapable tautology that these mental processes are the product of an individual's mind. A cognitivist perspective on creativity locates it in the person or more specifically in a person's head. A broad palette of instruments has been developed to measure creativity. Self-report measures range from assessing ratings of creative ideation to eliciting creative attitudes. Others, such as creative behaviour inventories, are more neutral with respect to the ontological locus of creativity. Performance tasks of divergent and convergent thinking are also employed to measure creativity.

In this chapter, I focus on so-called insight problems as a platform to assess creativity. A taxonomy of such problems has been offered (Weisberg, 1995): The common feature of these problems is that they resist, in naïve participants, the direct application of knowledge in long term memory to transform their start state into their normative goal state. Yet these problems vary: Some problems are formulated such as to encourage a misleading interpretation or activate a prepotent response that must be abandoned or inhibited; operators might not obviously come to mind although the problems are usually elementary riddles which can be solved with simple operators. Finally, some specify a goal state, but it is the manner with which to reach the goal state that is not obviously apparent. However, all insight problems are designed to create or compound a conceptual difficulty that must be overcome through the development of new ideas that bring the solution within a "mental look ahead horizon" (Ohlsson, 1984, p. 124, emphasis added). 
The current theoretical debate in the psychology of insight problem solving aims to explain this "mysterious leap of imagination" (Ohlsson, 1984, p. 120) in one of two ways. On one side are proponents of a special unconscious process, with semantic inferences the likely candidate (Ohlsson, 1992): These inferences result from the propagation of activation through networks of semantic elements that eventuate in hitherto unconnected paths, linking distant and disparate elements, and result in a new interpretation or conception of the problem. This new interpretation cues new operators and a new conceptual plane within which the solution can be grasped. On the other side are researchers (e.g., Weisberg, 2018; Fleck \& Weisberg, 2013) who argue that the (re)analysis of the problem elements is evinced through conscious, deliberate, and effortful processes that promote a sounder and more productive interpretation of the problem (the so-called business-as-usual view). Proponents of both positions agree that the potential for discovering the solution resides, as it were, in the agent working on the problem not unlike how stumpers ${ }^{1}$ are easily understood once a certain misleading assumption is abandoned. The bottom line is that, whether consciously or unconsciously, the reasoning agent bootstraps herself to a new plane of understanding and the leap of imagination is the result of a mental process. She has no need to act in, on or through the world to find her answer.

\section{Costing the Price of a New Idea}

Creativity involves a cost; resources must be invested to make something new. From the special processes perspective, the price for the journey is nil since the agent is the passive vessel sailing along undercurrents of semantic activation that may take her to a promising destination. From a gradualist business-as-usual perspective a new idea is the result of investments in cognitive capacity and ability: The more such resources are at an agent's

\footnotetext{
1 "A big brown cow is lying down in the middle of a country road. The streetlights are not on, the moon is not out, and the skies are heavily clouded. A truck is driving towards the cow at full speed, its headlights off. Yet the driver sees the cow from afar easily, and avoids hitting it, without even having to brake hard. How is that possible?" (Bar Hillel et al., 2018, p. 112). Answer: it's daytime.
} 
disposal, the more can be invested, and the more likely a new idea is produced. But crucially, it is difficult to examine the exact nature of those transactions because of the covert nature of the exchange. In other words, cognitive psychology deals in unobservables: The exact manner with which old ideas are transformed into new ones cannot be directly observed. This daunting challenge is tackled through different strategies. A psychometrics strategy is adopted by some researchers: They seek to establish the positive association between measures of cognitive capacity—such as working memory and intelligence — and solution rates with both insight and analytic problems (e.g., Chuderski \& Jastrzębski, 2018). Eye tracking is another strategy employed to pin down, at least, the allocation of attentional resources to different problem elements (e.g., Bilalić et al., 2021). Finally, verbal protocols are yet another method to identify the steps and hurdles that pave the road to a new idea (e.g., Fleck \& Weisberg, 2013). Even so, these strategies have shortcomings which mean that they only partially attend to the challenge. Correlational evidence cannot by itself help shed light on how a problem was solved (Vallée-Tourangeau \& Vallée-Tourangeau, 2020); eye tracking data are vulnerable to many interpretations (Orquin \& Holmqvist, 2018); and it is unclear the extent to which participants' meta-narrative reliably and validly maps onto mental processes (Ross \& Vallée-Tourangeau, 2021).

All scholarly and scientific enterprise should abstain from peddling tautological explanations. For example, the success of someone solving syllogistic reasoning problems may be attributable to intelligence. However, intelligence is commonly operationalised in terms of tests that involve syllogistic reasoning; hence what's on offer here is a circular nonexplanation. Science chisels the stuff that make up the world. The stuff is composed of substances which are sculpted through the gradual identification of attributes and actions. These substances are reified through tests and trials, and their behaviour noted. The goal of science is to understand how a substance emerges from a set of attributes and their actions (to 
adapt Latour, 2016). To explain creativity while avoiding tautology, one must therefore be careful not to draw upon attributes that have a creative pedigree of their own; there lurks an unproductive regress that begs the question and vitiates the explanation. The psychometrics strategy employed by some researchers in insight problem solving appears to avoid this trap by seeking to establish correlations between participants' cognitive skills and capacities with their performance on insight problems. The research programme typically proceeds by having participants work on a battery of insight problems from which an aggregate solution rate is computed. In the same testing session (these testing sessions can be quite long, spanning the better of eight hours, see Chuderski \& Jastrzębski, 2018) participants' cognitive capacity and skills are gauged through a battery of working memory span tests and tests of fluid intelligence. The data analysis strategy then calculates the correlation between aggregate performance on the insight problems and measures of working memory and intelligence. Typically, these correlations are positive. But the heavy cost of avoiding tautology is that this experimental procedure and analysis strategy are quite unable to explore the relationship between the microgenesis of new ideas and problem solution pathways. In other words, this research methodology does not and cannot help researchers understand the actual processes involved in the discovery of problem solutions. As such creativity remains unexplained.

The inability of the psychometrics manifesto to unveil creativity can be attributed to three key factors. First, performance on any given problem is lost to aggregate indices; it is not technically unrecoverable, but it is usually not reported. Second, the granularity of the analysis for performance on a given problem - the data capture - is insufficient to reveal how participants solved any given problem. Third, the research methodology employs so-called second order tasks, that is problems are presented as riddles in the form of propositions, rather than as first order tasks, that is with a physical model of the problem that can be manipulated and physically transformed (Vallée-Tourangeau \& March, 2019). First order 
problem solving requires interacting with the world, which alters the cognitive terrain (to adapt Kirsh, 2010, p. 443). Interactivity may scaffold thinking resulting in better performance, but and more important, interactivity makes the process visible. It lends itself to a careful recording of participants' actions, the resulting changes to the physical properties of the problem, and how these changes trigger new actions and perceptions. The data are rich and invite a granular qualitative mapping of the trajectory — in space and time - of the changes to the problem from start state to goal state. Equally important, this granular analysis can be applied to problem solving successes and failures symmetrically, unveiling conceptual breakthroughs as well as cul de sacs. It turns unobservable cognitive processes into observable actions and changes in the world (observables to participants as well as to researchers).

Let me wrap up these introductory remarks. Creativity does not materialize out of nothing: it involves an epistemic transformation which has a cost attached to it. Researchers should not lose sight of these costs and taking a loan on unconscious processes or intelligence is only a temporary measure: The loans should eventually be repaid. The exact nature of the transaction is difficult to inspect because of its covert nature. This difficulty is compounded by methodological decisions which reflect a mentalist ontological commitment; the disciplinary identity and concomitant professional exigencies naturally constrain the focus of the cognitive psychologist to look at the mind and limit the exploration of creativity to a mind that is artificially carved out of the body and the world in which it is embedded. Second order problem solving task environments can only be navigated mentally. In the absence of interactivity, no actions on the world can be recorded, no changes to a physical model of the problem can be captured, no trajectory from start state to goal state can be traced objectively. A psychometrics research programme, focussing exclusively on aggregate measures of performance does not unveil how new ideas arise. Working memory and intelligence may 
correlate with aggregate solution rates, but the origin of these solutions remains shrouded in a mysterious cloak.

\section{The Kinenoetic Field}

Cognitive archaeologists deal with objects produced by ancestral hominins and formulate conjecture on the nature of these hominins' cognitive abilities. Malafouris's material engagement theory $(2013,2020)$ introduces notions such as 'thinging' and the 'hylonoetic field'. These notions cast the association between objects and minds in transactional co-constitutive terms. The term 'hylonoetic' combines matter and knowledge, and was coined to capture the idea of cognition, cognitive development and evolution as proceeding with and through engagement with materiality. Take the symmetric shape of the oldest Acheulean handaxe: It has been argued that this reflects intentionality, the enactment of a mental plan in these ancient hominins' mind to create an artefact of that particular shape. Stone tools then are considered

"to be windows into prehistoric minds, not components of thinking itself. Assigning agency to hominins and using artifacts to document minds provided useful insights into the evolution of certain cognitive abilities, but it ignored the role that tools themselves played in early hominin cognition, and how that role influenced the evolution of cognition itself." (Wynn et al., 2021, p. 101).

In contrast, and from a material engagement perspective, the features of stone tools, such as handaxe symmetry, emerge out of the manufacturing process:

"The stone projects towards the knapper as much as the knapper projects towards the stone and together they delineate the cognitive map of what we may call an extended intentional state. The knapper first thinks through and 
with the stone before being able to think about the stone and hence about one's self as a conscious reflectively aware agent." (Malafouris, 2010a, p. 19).

Symmetry is a by-product of engaging with the materiality of the flint and the hammer stone rather than its cause. This is a particularly fruitful perspective on innovation and tool making because it takes no loan on the conjectured representational or intentional abilities of these hominins. Rather, it casts intelligent innovation as a function of engaging with the world, of manipulating and transforming objects. Innovation and creativity emerge out of the concurrent transformation of the so-called subject and object. Innovation here is explained not in terms of an innovative mind and eschews the regress entirely by relying on processes that do not presume innovation but out of which it emerges. These dynamically unfolding object-thought mutualities are, however, themselves conjectures because their co-evolution cannot be directly observed: the objects are archaeological artefacts, the temporal scale epochal, their creators long silent.

Fast forward several millennia to a cognitive psychology laboratory. The aim of the lab is not to conject but to establish how a naïve participant discovers the solution to an insight problem. The problem description is accompanied by a manipulable object, an object that can be made into a physical model of the solution. To be sure, the creative innovation is not of the same magnitude or interest as those reflected in the manufacture and manipulations of important artefacts (e.g., such as the Mycenaean Linear B tablets; Malafouris, 2010b) and the inferences about the creative and innovative potential of ancestral hominins is a taller challenge than those of a typical university undergraduate participating in a problem-solving experiment. Nonetheless, a properly instrumentalized procedure can reveal the transactional co-constitutive evolution of the participant's understanding of the solution and the physical model of the problem. In other words, object-thought mutualities can be directly observed. 
The movement or transformation of the object both reflect and trigger change in the participant's understanding of the problem. New ideas from this perspective emerge from a kinenoetic field, a term that underscores the importance of object transformations in space and time (see Ross \&Vallée-Tourangeau, under review). These transformations are not perceived passively, they are not traced through binoculars by a distant observer. The movements result from the agent-world coupling. The relief of the kinenoetic field is mapped by recording the dynamic changes in the nature and position of the object.

Let me shift the narrative to a more concrete register with a classic visuo-spatial insight problem, the triangle of coins (top of Figure 1). In this problem, participants must discover how to transform a configuration of 10 coins in the shape of a triangle that points down to one that points up. The difficulty of this problem does not lie in the nature of the operators, nor in how the goal state should look like since imagining the shape pointing up requires elementary visuo-spatial skills and corresponds to a well-encoded and frequently encountered cultural object or symbol. The challenge lies in the following constraint in the problem definition: The goal state is realized by moving three and only three coins. Thus, the new idea that is required, the new thought that needs to be constructed, is wrought through the identification of the critical coins among the set of 10. Participants work to discover which three coins must be moved to change the shape of the triangle. The critical coins are the vertices, colour coded in Figure 1 (although not for participants working on the problem); the solution is illustrated in the top right panel of Figure 1. Few participants solve this problem quickly, with a substantial proportion failing to find the answer within 10 minutes. 

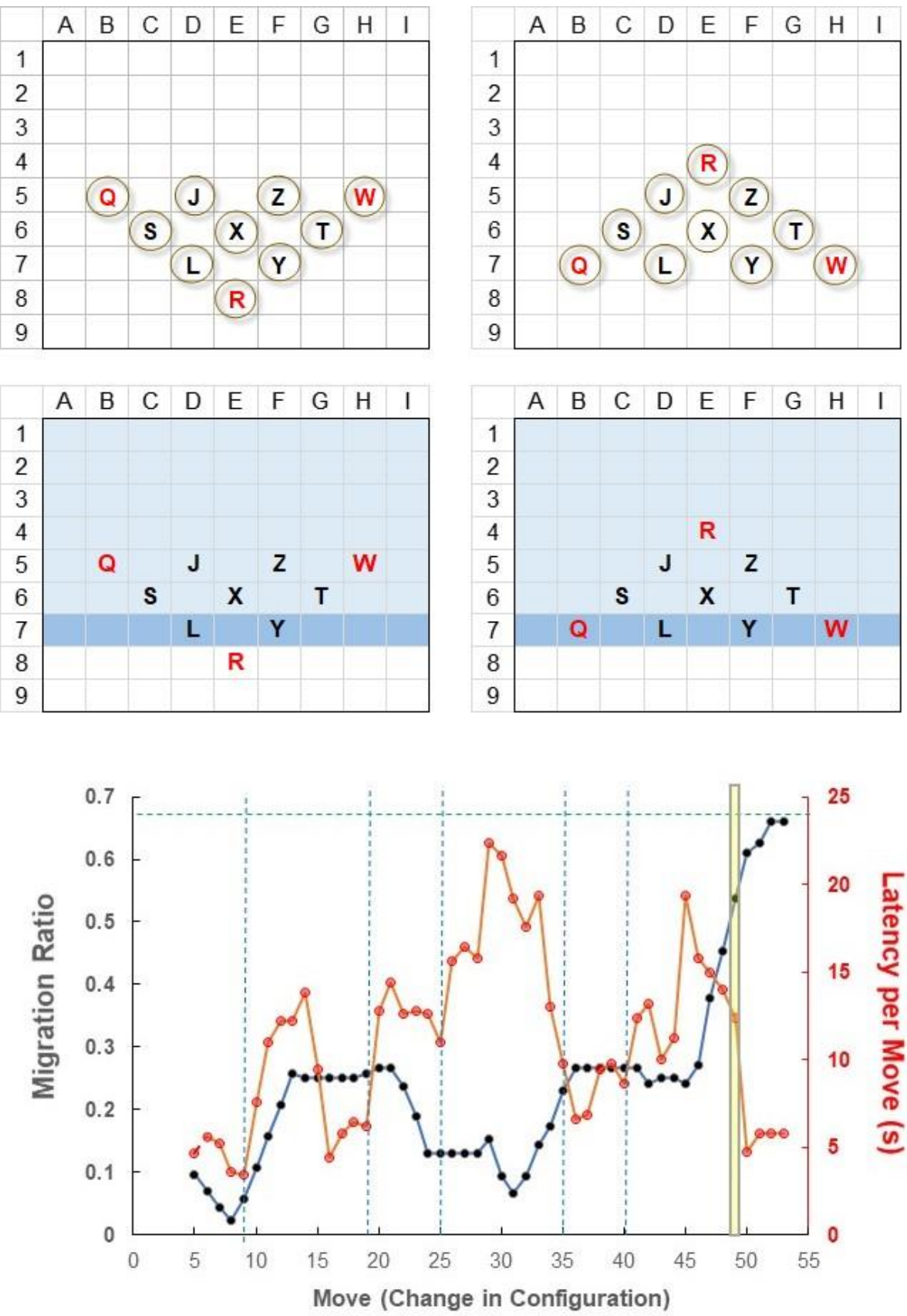

Figure 1. Top panels: start and goal state of the triangle of coins problem; vertices are colour coded here for illustrative purposes, but the coins were not colour coded in this manner for participants. Middle panels illustrate the migration ratio; number of coins along row 7 divided by the number of coins in the rows above (.29 at the start state, .67 at the goal state). Bottom panel: Migration ratio (primary y axis) and latency per move (secondary y axis) for a participant working and solving the triangle of coins (adapted from Vallée-Tourangeau et al., 2021), plotted as running average over the previous five moves. 
We instrumentalized the experimental procedure in the following manner (ValléeTourangeau et al., 2021). First, we present the problem as a first order one, that is with a physical model of the problem where participants can move the coins and observe the results of their moves, namely the new configuration that is produced by the movements. The problem is presented as an interactive game on a computer tablet with a reset button and participants can reset the board as often as they wish to its initial configuration, as a triangle pointing down. Second, we labelled the coins and arrayed them on a $9 \times 9$ grid with numbered rows and lettered columns. Third, we filmed participants as they worked on the problem and it is this video evidence that informed a detailed granular mapping of the moves and the change in the object over time. Instrumentalizing the procedure in this manner coupled with the video evidence, help us recover — and with precision — which coin was moved where and when, and capture the iterative relationship between the configuration of the problem and the participants' actions. It is this iterative relationship that is responsible for producing, what we are calling, "object-thought mutualities" and our videoed, instrumentalised procedure allows us to re-create, for each participant a chart of the temporal-spatial kinenoetic field. In addition, we can animate the movements, an analytic strategy that clearly reveals the types of configuration that are repeatedly explored, those that are abandoned, and the trajectory to the goal state. One such animated trajectory for one of our participants can be accessed at https://osf.io/et4pq/: It recreates the dynamic changes to the problem configuration as the participant labours to discover the identity of the three critical coins - the vertices - to evince a solution. The animation also illustrates the cost of discovery, the slow, non-linear, and heteroscalar evolution from a state of unknowing to a state of knowing; it captures the genesis of a new idea.

In addition to this animated reconstruction of the trajectory, we created an index we call the "migration ratio". The migration ratio indicates numerically the extent to which changes 
in the resulting configuration approximate the target configuration (the solution). The middle panels of Figure 1 illustrate how the migration ratio is calculated. It is the ratio of coins along row 7 (the dark blue row) which will form the base of the correct triangular configuration over those in all the rows above (light blue rows). At the start state, the migration ratio is $2 / 7$ or .29; at the goal state it is $4 / 6$ or .67 . In addition to creating a map of the kinenoetic field, we can create another inscription device that illustrates how coins are gradually configured to approximate the target migration ratio. The migration ratio can capture efforts to move coins down to form a base along row 7, rather than migrating coins north, a common early strategy observed with this problem (in the above cited video animation this is clearly observed for the first 45 moves that aim to move coins north without migrating the base of the triangle down). The bottom panel of Figure 1 plots the migration ratio (along the primary y axis) and the latency per move (along the secondary y axis) as running averages over the previous 5 moves. The dynamic unfolding of these data series is also illustrated in the animated video.

This inscription device provides compelling perceptual evidence of two key features of the process of discovering the critical coins with which the normative solution can be constructed: (i) it is gradual, as reflected by the rise in the migration ratio over the last 10 moves; and (ii) it is heteroscalar, the process unfolds along different time scales, as reflected by the oscillation in move latencies. The gradual rise of the migration ratio graphically demonstrates that the participant does not know the answer before it is physically constructed in front of her. This is also confirmed by the participant making an incorrect announcement illustrated by the strip of highlighted yellow in the bottom panel of Figure 1 (at this point she had moved four coins to create a triangle pointing up); after receiving feedback that her solution was incorrect - the feedback was generic, right or wrong, without specifying which coins were in the correct or incorrect position — she created the right configuration and then announced her answer. Note that participants were not forced to physically create the right 
configuration, that is they could announce their solution at any time, on the basis of mental projections. However, with one exception, all our participants constructed the right configuration and then announced the solution (Vallée-Tourangeau et al., 2021).

The fact that all our successful participants laboured to construct the solution before announcing it implies an iterative transactional process between what the agent knows and the physical realization of the solution. In other words, these object-thought mutualities reveal the co-determination of epistemology and ontology. Until the solution is constructed, knowledge about the object and the nature of the object are in a relationship of unstable codependence which finds equilibrium and sediments only as the object's normative shape is constructed. March and Malafouris (this volume) describe this process of becoming as "learning into existence". The solution is materially enacted rather than being mentally simulated first and then physically implemented. This suggest that for some participants, insight should be better thought of in terms of 'outsight' (Vallée-Tourangeau \& March, 2019): and indeed some of our participants exhibited stereotypical eureka moments - markers normally associated with a phenomenological response to insight —after seeing the constructed solution but not before (see https://youtu.be/ZZSC549UyTg, note at 0:00:07 the double-take expressed with both hands splayed open when the participant created the solution).

\section{Insight Reductionism and the Deceitful Inversion}

Insight is classically defined as a sudden gestalt, marking the swift reorganization of a mental representation (Gilhooly \& Webb, 2018). In Fleck and Weisberg's (2013) integrative framework developed from extensive verbal protocol data, insight, the pure kind - that is the distinct phenomenological experience that arises after a protracted moment of impasse and accompanies the sudden appearance of a new idea - is relative rare. In their study, these pure 
insight moments accounted for $7 \%$ of the solutions in the verbal protocols. In Danek et al. (2020), $15 \%$ of the correct solutions occurred suddenly, and these sudden solutions were coupled to higher Aha! ratings.

The paucity of the phenomenon does not stop researchers from better understanding it. Efforts are made to triangulate it through different methods, including brain imaging as well as interventions to encourage a less controlled form of thinking (e.g., hypofrontality, the transient or neuropsychologically chronic kind; Dietrich, 2019; de Souza et al., 2014) by weakening executive function. These efforts are sometimes prefaced with vignettes of discovery as reported by the thinkers or innovators who claim to have had new ideas of momentous importance in a dream or while engaging in unrelated pedestrian activities.

As Brannigan (1981) argues, the explanatory or the "causal adequacy of the gestalt switch" as a model of the origin of a new idea is questionable. The gestalt switch explanation "[i]n its simplest expression (...) takes the form of a recommendation that discoveries occur as the result of gestalt switches. Since the switch is the thing that is occurring, it is circular to identify it as a cause of this" (p. 36). Significantly our first order approach provides compelling evidence that the gestalt switch can trip after the solution is constructed.

Betting on gestalt switches, we, as others have argued, will not pay off ${ }^{2}$. As a psychological phenomenon, such switches deserve attention (e.g., Danek, 2018); however, elevating the phenomenological experience either as a marker of a process (see Ross, 2021) or as a cause of creativity and discovery does not pave the way for a productive programme of research. Investing explanatory capital in gestalt switches buffers the theoretical retreat against other explanations of creativity in terms of microprocess that operate at the agent-

\footnotetext{
${ }^{2}$ In the experiments with the triangle of coins reported in Vallée-Tourangeau et al. (2021) self-report measures along four dimensions (happiness, suddenness, relief and certainty) were recorded from participants who announced a correct solution. Ratings along the suddenness dimension were significantly lower than the other dimensions. There was little evidence that the successful participants experienced a sudden shift in comprehension.
} 
world interface. We turn away from the notion of insight and look instead to Latour and Woolgar (1986, Chapter 4 "The microprocessing of facts") and their critical reflections on the 'special' nature of scientific thinking in contradistinction to any other form of thinking:

"[in stopping the] sociological enquiry at the level of mute individual thought (...) science will once again appear extraordinary. Our position is not unlike the opponents of vitalism in nineteenth-century biology. No matter what progress was made by biologists to explain life in purely mechanistic and materialist terms, some aspects always remained unexplained. There were always some corners in which notions of "soul" or the "pure vital force" could find refuge. Similarly, the notion that there is something special about science, something peculiar or mysterious which materialist and constructivist explanations can never grasp, is pushed further and further. But this notion will remain as long as the idea lingers that there is some peculiar thinking process in the scientist's mind. It is to complete our argument and to hamstring efforts to rescue the exotic view of science that we need tentatively to embark upon this new level of microprocessing." (p. 168)

To kindle the faint promise of the insight phenomenology as a marker and cause of creative ideation, the research programme proceeds primarily with task environments that do not involve participants interacting with a physical model of the problem. This methodological decision is driven by an ontological commitment to an internal locus of creativity (namely in the head), as well as the search for a single cause, a singular moment in space and time that explains the discontinuity from not knowing to knowing. But this discontinuity is not a salto mortale, the unbridgeable gap between routine and non-routine cognition (Ohlsson, 2018), it is not a mysterious leap. The distributed and protracted nature of creativity — which in turn problematizes notions of agency and intentionality - is apparent 
in qualitative studies of artists, scientists and designers (Glăveanu et al., 2013; Ross \& Vallée-Tourangeau, 2021; Vallée-Tourangeau \& March, 2019; the gradual protracted nature of scientific discovery is well illustrated in Woolgar, 1988) and calls for an entirely different explanatory model of creativity, not a reductionist one that leaps upon a single, distinct, linear cause, but a more historically and materially embedded, systemic and descriptive model of creativity.

Changes in the world brought through actions on objects, trigger new perceptions and afford actions that pave a creative ideation trajectory. This trajectory is a route without a road: The route to the solution is not traced in advance, it is not a route to be discovered, it is a route that is enacted. The theoretical effort needed to make sense of this interactivity ought to warn us of the dangers of falling prey to a default Cartesianism that so easily seems to fit folk and subjective notions of thinking and agency (apparent in the retrospective narrative of the trajectory of an idea; “one day so and so had an idea", see Latour \& Woolgar, 1986, pp. 169-171). The solution is twofold. First, we must not lose focus on the transactional nature of the interaction: agent and object are co-constituted through interaction. This encourages a shift from an implicitly dualist programme of research to one that casts agent-object as a system, a dynamic and contingent one. Second, the nature of the explanation of creative ideation should be unreservedly and unashamedly descriptive and historical without diminishing the scientific rigour of the account. As Sutton (2010, p. 214) writes: "we shouldn't work with an overly restricted or puritanical notion of scientific explanation: nonpredictive narrative explanations are common enough in the natural and social sciences of many complex systems, including branches of history, geography, geology, evolutionary biology, and meteorology." Stengers (1997, p. 171) speaks of a Darwinian narrative, not 
simply applied to the evolution of organisms, but to a historical narrative that explores "the diversity of causes and the diversity of ways in which the same cause can cause ${ }^{3} . "$

The detailed mapping of the kinenoetic field is a labour of granular description of actions and changes in the world. The kinenoetic field is not an irresistible cornucopia of good ideas: it maps out a trajectory, the paths that lead to solution as well as the unproductive directions along which the system evolves to failure. In other words, this granular description treats successes and failures symmetrically: revealing equally how participants' actions reinforce unproductive configurations as well as how they can reveal more productive paths to solution. The animated video of a participant working on the triangle of coins presented earlier illustrates that the participant at some point abandons migrating coins north and start migrating coins south which will eventuate in constructing a wider base along row 7 which is the key to the solution. It is tempting to identify this as a pivotal moment in the trajectory. Why did the participant choose this new strategy? (Note how the language is imbibed in agency and mental plans: the words 'choose' and 'strategy' make the resistance to an agentic and dualist account of creative ideation difficult even if the 'why' is not clear and we have no way of knowing the exact reason for the shift in action.) Hindsight makes the question seductively tempting. The participant solved the problem, and this new pattern of coin migration invites the attribution of the success to this change in migration pattern. But this question is not helpful and injects some life in a dormant dualism. We know that the participant solved the problem, and it is tempting to seek a key pivotal switch in thinking and action that sent the participant along the 'right' path. It's an abductive thought reflex, the best explanation for the success of the participant is attributing foresight (cf. Ingold, 2010). The

\footnotetext{
${ }^{3}$ Stengers (1997, pp.171-172) continues: "Darwinian authors are thus neither judges, poets, nor prophets, because the history of life as they have learned to read it does not authorize any principle of economy, that is, it does not permit the invention of the relationship of forces that would allow an object to be created and judged, or a hierarchy of questions to be established."
} 
inversion creeps in at this stage ${ }^{4}$ : knowing that the participant has solved the problem, we seek to retrospectively coronate an event pivot, endowing it with causal power that it does not have. We must resist this way of analysing creativity. A gold medallist in the 100-metre freestyle might have loved swimming at the age of four, but her childhood enthusiasm and precocious talent do not explain her achievement: no single cause can be identified other than the careful description of the training and coaching unfolding in the thousands of hours of deliberate practice 5 .

The creative trajectory, and in the case of insight problem solving the trajectory to the normative solution, is constructed with contingent microsteps. The dual pull of looking for a cause and looking for it inside the agent's head will forever blind researchers of creative ideation to its developmental and systemic trajectory and validate an unproductive form of reductionism. Latour (2016) contrasts two types of reductionism. The first was alluded to earlier: the felicitous type of reductionism attempts to explain creativity from a set of actions out of which it emerges. It naturally encourages a descriptive ethnographic and historical account of the interaction with objects ("it makes you friends of interpretable objects"; Latour, 2016, p. 96), mapping in granular details, actions and object transformations in time

\footnotetext{
${ }^{4}$ de Vries (2016, p. 34) sums up one of the main lessons of Latour and Woolgar's (1986) Laboratory life: Once all modalities in qualifying a statement about the world are dropped “(...) a fact has been established. It is accepted that 'a mammal injected with a substance with chemical structure XYZ will show an enhanced form of $\mathrm{ABC}$ behaviour'. At this point, an important inversion has taken place (...) history will be rewritten by inverting process and outcome. From this point on, the process will be narrated as the search for this particular outcome: this is the fact that the scientists have been looking for. It took quite some effort before it was discovered, but by now its existence has been revealed. From now on, the history is perceived from this vantage point: the process of scientific discovery is turned into the pursuit of a single path which inevitably led to the discovery of the structure of this substance (...)"(emphasis added).

${ }^{5}$ Sir Dave Brailsford explained the extraordinary success of British cycling in terms of the cumulative effects of small changes to features of the entire system: "By experimenting in a wind tunnel, we searched for small improvements to aerodynamics. By analyzing the mechanics area in the team truck, we discovered that dust was accumulating on the door, undermining bike maintenance. So we painted the door white, in order to spot any impurities. We hired a surgeon to teach our athletes about proper hand-washing so as to avoid illnesses during competition (we also decided not to shake any hands during the Olympics). We were precise about food preparation. We brought our own mattresses and pillows so our athletes could sleep in the same posture every night. We searched for small improvements everywhere and found countless opportunities. Taken together, we felt they gave us a competitive advantage." From Harrel, E. (2015). How 1\% performance improvements led to Olympic gold. Harvard Business Review, October 2015. http://thebusinessleadership.academy/wpcontent/uploads/2017/03/How-1-Performance-Improvement-Led-to-Olympic-Gold.pdf
} 
and space. You can account for creativity in this manner by illustrating its distributed and protracted nature. The second type of reductionism prevents such accountability, curtails the detailed ethnographic mapping of the unfolding of creativity by steamrolling over "the list of actions of which it is no longer a summary but now the source" (p. 96). At play here is a deceitful inversion: the end result is cast as the cause of the process that brought it to light. From Latour's lectern, this form of reductionism is "the bane of science" (p. 96).

\section{Concluding Remarks}

Problem solving researchers who enforce and validate the limits of creativity research by working exclusively on second order problem solving tasks - tasks that can only be solved mentally — end up conjecturing exactly what they are looking for, namely that new thoughts are evinced through mental processes. Such a research strategy, based as it is upon unobservable mental shifts, is at risk of producing explanations that are analytically empty.

First order problem solving proceeds with and through the world. Since the world is there to see and touch, things can be physically transformed and perceptually seized, the representation cost and burden are alleviated considerably. The restructuring so fondly postulated as an explanation of representational change can proceed in the world rather than (exclusively) in the head (Vallée-Tourangeau, 2014). A first order problem solving procedure opens the field of research by problematizing agency and instrumentality. It admits a broader class of external events that contribute to the changes in the objects, including microprocesses of a serendipitous nature (Ross \& Vallée-Tourangeau, 2020). These events do not undermine a 'psychological' account of creativity as some fear (see Chuderski et al., 2020), they expand it, and in the process align it more closely with the practices of artists and scientists (Glăveanu et al., 2013). 
A first order task environment such as the laboratory procedure described earlier with the triangle of coins offers the opportunity to chart the transformation of an object that reifies a model; in insight problem solving that object is a proto model of the solution. The research procedure maps, in space and time, the transformation of that object. The mapping efforts create new inscription devices that expose these changes (as illustrated in the animated trajectory to solution presented earlier) and these devices can be used as a communication tool with other members of the research community. The complexity of creativity is mobilized in the laboratory and its physical traces in turn reified through these inscription devices. The physical traces can be interrogated and analysed, helping the researcher see the unproductive explorations as well as those that potentially pave the way to discovery. The gauntlet of the unobservables is replaced with the patient qualitative description of observables.

The account that is formulated from this qualitative analysis runs the risk of being misunderstood as a reversal of agency with problem solvers being turned into puppets at the mercy of an external environmental puppeteer, namely things in the world, but this a false fear born out of deep rooted Cartesianism. It is more productive to cast agency and intentionality as extended and distributed, as emerging features of the agent-world coupling that configure a system of resources, thoughts and affordances. Researchers employing first order task environments can bootstrap their explanation of creativity out of the tautological trap that closes on accounts formulated in terms of intelligence or individual differences measuring creativity. It is not simply that the loan on intelligence is repaid; no mortgage application is submitted in the first place.

The kinenoetic field can only be mapped using an interactive problem-solving procedure; a task environment that allows and encourages an engagement with a physical model of the solution. In addition, the procedure must be instrumentalized to allow the 
recording of the spatial and temporal features of these transformations. Researchers must invest time to produce a granular qualitative description of the participants' actions and the resulting changes to the physical model of the problem. These efforts map out the kinenoetic field from which a new idea emerged, unveiling the nature and cost of the transaction that purchased a new idea. It is the dynamic co-constitutive coupling of agent and world that evinces a new idea. 


\section{References}

Bar-Hillel, M., Noah, T., \& Frederick, S. (2018). Learning psychology from riddles: The case of stumpers. Judgment and Decision Making, 13(1), 112-122.

Bilalić, M., Graf, M., Vaci, N., \& Danek, A. H. (2021). The temporal dynamics of insight problem solving - restructuring might not always be sudden. Thinking \& Reasoning, 27(1), 1-37. https://doi.org/10.1080/13546783.2019.1705912

Brannigan, A. (1981). The social basis of scientific discoveries. Cambridge University Press.

Chuderski, A., \& Jastrzębski, J. (2018). Much ado about aha!: Insight problem solving is strongly related to working memory capacity and reasoning ability. Journal of Experimental Psychology: General, 147(2), 257-281. https://doi.org/10.1037/xge0000378

Chuderski, A., Jastrzębski, J., \& Kucwaj, H. (2021). How physical interaction with insight problems affects solution rates, hint use, and cognitive load. British Journal of Psychology, 112(1), 120-143. https://doi.org/10.1111/bjop.12442

Danek, A. H. (2018). Magic tricks, sudden restructuring and the aha! experience. In F. Vallée-Tourangeau (Ed.), Insight: On the origin of new ideas (pp. 51-79). Routledge.

Danek, A. H., Williams, J., \& Wiley, J. (2020). Closing the gap: Connecting sudden representation change to the subjective Aha! Experience in insightful problem solving. Psychological Research, 84, 111-119. https://doi.org/10.1007/s00426-018-0977-8

de Souza, L. C., Guimaraes, H. C., Teixeira, A. L., Caramelli, P., Levy, R. et al. (2014). Frontal lobe neurology and the creative mind. Frontiers in Psychology, 5, 00761. https://doi.org/10.3389/fpsyg.2014.00761

de Vries, G. (2016). Bruno Latour. Wiley. 
Dietrich, A. (2019). Types of creativity. Psychonomic Bulletin \& Review, 26(1), 1-12. https://doi.org/10.3758/s13423-018-1517-7

Fleck, J. I., \& Weisberg, R. W. (2013). Insight versus analysis: Evidence for diverse methods in problem solving. Journal of Cognitive Psychology, 25(4), 436-463.

\section{https://doi.org/10.1080/20445911.2013.779248}

Gilhooly, K., \& Webb, M. E. (2018). Working memory and insight problem solving. In F. Vallée-Tourangeau (Ed.), Insight: On the origins of new ideas (pp. 105-119). Routledge.

Glăveanu, V. P., Lubart, T., Bonnardel, N., Botella, M., Biaisi, P.-M. A. et al. (2013). Creativity as action: Findings from five creative domains. Frontiers in Psychology, 4; 176. https://doi.org/10.3389/fpsyg.2013.00176

Ingold, T. (2010). The textility of making. Cambridge Journal of Economics, 34, 91-102. https://doi.org/10.1093/cje/bep042

Kirsh, D. (2010). Thinking with external representations. AI \& Society, 25, 441-454. https://doi.org/10.1007/s00146-010-0272-8

Latour, B. (2016). How better to register the agency of things (Part 1, Semiotics). Tanner Lectures, Yale, March 2014, published in The Tanner lectures on human values, volume 34 (pp. 81-99). The University of Utah Press.

Latour, B., \& Woolgar, S. (1986). Laboratory life. The construction of scientific facts (second edition). Princeton University Press.

Malafouris, L. (2010a). Knapping intentions and the marks of the mental. In L. Malafouris \& C. Renfrew (Eds.), The cognitive life of things: Recasting the boundaries of the mind (pp. 13- 22). Cambridge: McDonald Institute Monographs. 
Malafouris's (2010b). Metaplasticity and the human becoming: Principles of neuroarchaeology. Journal of Anthropological Sciences, 88, 49-72

Malafouris, L. (2013). How things shape the mind: A theory of material engagement. MIT Press.

Malafouris, L. (2020). Thinking as 'thinging': Psychology with things. Current Directions in Psychological Science, 29, 3-8. https://doi.org/10.1177/0963721419873349

Ohlsson, S. (1984). Restructuring revisited II: An information processing theory of restructuring and insight. Scandinavian Journal of Psychology, 25, 117-129. https://doi.org/10.1111/j.1467-9450.1984.tb01005.x

Ohlsson, S. (1992). Information-processing explanations of insight and related phenomena. In M. T. Keane \& K. J. Gilhooly (Eds.), Advances in the psychology of thinking (Vol. 1, pp. 1-44). Harvester-Wheatsheaf.

Orquin, J. L., \& Holmqvist, K. (2018). Threats to the validity of eye-movement research in psychology. Behavior Research Methods, 50(4), 1645-1656. https://doi.org/10.3758/s13428-017-0998-Z

Ross, W. (2021). On the trail of a thought: A kinenoetic analysis of problem solving. Unpublished $\mathrm{PhD}$ dissertation. Kingston University.

Ross, W., \& Vallee-Tourangeau, F. (2021). Kinenoetic analysis: Unveiling the material traces of insight. Methods in Psychology, 5; 100069. https://doi.org/10.1016/j.metip.2021.100069

Ross, W., \& Vallée-Tourangeau, F. (2020). Microserendipity in the creative process. Journal of Creative Behavior, 55(3), 661-672. https://doi.org/10.1002/jocb.478

Stengers, I. (1997). Power and invention. University of Minnesota Press. 
Sutton, J. (2010). Exograms and interdisciplinarity: History, the extended mind, and the civilizing process. In R. Menary (Ed.) The extended mind (pp. 189-225). MIT Press.

Vallée-Tourangeau, F. (2014). Insight, materiality and interactivity. Pragmatics \& Cognition, 22(1), 27-44. https://doi.org/10.1075/pc.22.1.02val

Vallée-Tourangeau, F., \& March, P. L. (2019). Insight out: Making creativity visible. Journal of Creative Behavior, 54(4), 824-842. https://doi.org/10.1002/jocb.409

Vallée-Tourangeau, F., Ross, W., Ruffatto Rech, R., \& Vallée-Tourangeau, G. (2021).

Insight as discovery. Journal of Cognitive Psychology. 33(6-7), 718-737. https://doi.org/10.1080/20445911.2020.1822367

Vallée-Tourangeau, F., \& Vallée-Tourangeau, G. (2020). Mapping systemic resources in problem solving. New Ideas in Psychology, 59; 100812. https://doi.org/10.1016/j.newideapsych.2020.100812

Weisberg, R. W. (1995). Prolegomena to theories of insight in problem solving: A taxonomy of problems. In R. J. Sternberg, \& J. E. Davidson (Eds.), The nature of insight (pp. 157-196). MIT Press.

Weisberg, R. W. (2018). Insight, problem solving, and creativity: An integration of findings. In F. Vallée-Tourangeau (Ed.), Insight: On the origins of new ideas. (pp. 191-215). Routledge.

Woolgar, S. (1988). Science: The very idea. Ellis Horwood Tavistock Publications.

Wynn. T. Overmann, K. A., \& Malafouris, L. (2021). 4E cognition in the Lower Paleolithic. Adaptive Behavior, 29(2), 99-106. https://doi.org/10.1177/1059712320967184 